\title{
Birth Asphyxia at a District Hospital in Kigali, Rwanda
}

\author{
Fauste Uwingabire ${ }^{1 *}$, Marcella Gowan ${ }^{2}$ \\ ${ }^{1}$ School of Nursing and Midwifery, University of Rwanda, College of Medicine and Health Sciences, Kigali, Rwanda \\ ${ }^{2}$ University of Illinois at Chicago, Linfield College, Good Samaritan School of Nursing, USA
}

*Corresponding author: Fauste Uwingabire. School of Nursing and Midwifery, University of Rwanda, College of Medicine and Health Sciences, Remera Campus, 11 KG 47, Kigali, Rwanda. Email: uwingabirefauste@gmail.com

\begin{abstract}
Background

Prematurity, birth asphyxia (BA), and sepsis are the leading causes of neonatal mortality. The BA prevalence rate is $23 \%$ worldwide and affects up to $39 \%$ in Rwanda. Birth asphyxia is an insult with significant consequences to the neonate's brain and other vital organs due to decreased oxygen before, during or immediately after birth.

Objective

To assess the BA prevalence, associated factors, and outcomes at a district hospital in Kigali. Methods

A retrospective, cross-sectional study of 340 neonates admitted to the NICU in 2016.

\section{Results}

Birth asphyxia was identified in 135 out of 340 newborns (39.7\%). Associated factors included gravida, meconium-stained amniotic fluid, normal duration of labor, normal duration of ROM, vaginal delivery, Apgar score of $\leq 5$ at 5 minutes, respiratory ventilation and cardiac massage and normal birth weight (2500-3999gr). Two-thirds were born via vaginal birth (66.5\%), and nearly half $(47.3 \%)$ had BA. One-third was born via cesarean $(33.5 \%)$, and a quarter $(24.6 \%)$ had BA. Other BA outcomes included prolonged hospitalization beyond the neonatal period at $75 \%$ compared to non asphyxiated babies (25\%). The mean days of NICU stay was 7.6 (SD: 6.1) for BA while it was 5.3 (SD: 4.16) for non-asphyxiated babies; early seizures (52.6\%) and high specific mortality $(87 \%)$.
\end{abstract}

\section{Discussion}

Findings revealed a $6 \%$ BA prevalence increase, and a BA-specific mortality rate 13 times higher than previously recorded. Trained healthcare professionals need to reverse this significant trend in both vaginal and cesarean births that likely has a profound effect on the neonate, family, and community.

Rwanda J Med Health Sci 2019;2(2):96-104.

Keywords: Birth asphyxia, neonatal morbidity, mortality, prevalence, risk factors, outcomes, subSaharan Africa

\section{Background}

The three leading causes of neonatal mortality worldwide are prematurity, intrapartum/birth asphyxia (BA), and neonatal sepsis.[1] The neonatal period is marked by significant vulnerability for the neonate, defined as the first 28 days after birth.[1]In 2017, there were 7,000 deaths per day, with an annual mortality rate of 2.5 million, and the majority of deaths within the first week after birth.[1,2] An additional 2.6 million were stillborn. $[1,2]$ Birth asphyxia is caused by an interruption of blood flow and gas exchange to the fetus, and if severe, can lead to hypoxic-ischemic encephalopathy (HIE) and resultant demise or long-term neuronal complications. [3] However, in many cases, BA is preventable with high-quality evidenced-based interventions. [4,5]
Neonatal deaths and stillbirths have declined at a slower rate than other under- 5 deaths. $[6,7,8]$ Therefore, more concentration is needed on the neonatal population, which is part of Sustainable Development Goals (SDG), specifically (SDG 3.2). Birth asphyxia, perinatal asphyxia, and neonatal asphyxia are all terms referring to the same neonatal complication of a hypoxic event before, during or shortly after birth.[4,9,10,11] Birth asphyxia is not just a medical diagnosis, but also a nursing and midwifery concern, as the standard neonatal care after birth is prompt drying, stimulation and warmth, which is sufficient intervention to initiate and maintain breathing for $99 \%$ of neonates. $[12,13,14]$ A recent audit in Rwanda indicated $60 \%$ of deaths were potentially preventable.[15] 
In Rwanda, the Ministry of Health $(\mathrm{MOH})$ reported that BA accounted for $39 \%$ of neonatal deaths in 2013,[16] and 41\% in 2014.[17]Based on these statistics we know the national BA mortality rate, but unaware of the risk factors and information on what happened to the neonate after surviving birth and admitted to the neonatal intensive care unit (NICU). This study provided the much-needed data on BA from a busy district hospital in Rwanda's capital city of Kigali.

BA is among the leading causes of neonates' admission to the NICU and still causing neonatal deaths worldwide. $\mathrm{BA}$ was reported as the cause of $16.2 \%$ of NICU admissions in Ghana,[18] 12.5\% in Ethiopia,[19] 22\% in Uganda,[20] and $51.3 \%$ in Nigeria.[4] Findings from studies in other continents have a similar wide prevalence range. The outcomes of neonates admitted to the NICU with BA include motor and cognitive disabilities, and early neonatal death (within first 24 hours).[10,11,21]

Our study was guided by the conceptual framework of Magadi and colleagues,(2004).[22] The model was adapted from the current literature to fit the Rwandan context. The following variables were included: maternal factors(age, ANC visits, gravida, and co-morbidities); fetal factors (gender, birth weight, gestational age, multiple births, IUGR);placental factors (placenta, cord); and labor, birth, and post-natal factors (prolonged labor, amniotic fluid, mode of delivery, dystocia, Apgar score, cry at 5 minutes, and resuscitation).The measurable outcomes of BA included; discharged home, referred to another hospital, or demise. The aim of this study was to assess the prevalence, associated risk factors and outcomes of birth asphyxia in a NICU at a district hospital in Kigali.

\section{Methods}

\section{Design}

A descriptive, retrospective, cross-sectional study of 340 neonates admitted to a district hospital NICU during 2016. The 160-bed maternity hospital in Kigali was selected due to the high volume of births and admissions to the NICU. There was an average of 25 births a day, or about 750 per month, and an average occupancy rate of $78 \%$ in the main hospital. It was a public hospital that received patients with public and private health insurance, and self-pay patients. As a district hospital, it served as a referral site for 10 health centers, and a clinic at the central prison of Kigali. Eight of these health centers provided maternity services, conducted normal (low-risk) births and referred highrisk labors, and complicated post-partum and postnatal cases to the district hospital for further management.

In 2016, the hospital NICU consisted of 10 incubators, six radiant warmers, and 10 beds for Kangaroo Mother Care (KMC) and 10 cots. One medical doctor, five midwives, and six nurses provided care $24 / 7$. The NICU provided observation, intensive care, high dependent care, KMC, and neonatal monitoring, until the neonate met the criteria for discharge, referred to a tertiary level facility, or died.

\section{Sample}

In 2016, there were 1441 neonates admitted to the NICU from 1 January to 31 December, and this number constituted the study population. All neonates born at the district hospital, or born outside the hospital and admitted to the NICU for any medical reason, were randomly selected to be included in the study population.

Preterm neonates less than 35 weeks gestation, unknown gestational age, and those with congenital defects were excluded. Other excluded cases were neonates admitted to the NICU without a medical diagnosis or a mixed like those with social issues (abandoned babies, those brought in by the police, mothers had died or transferred to another facility, or those lacking family support).

The sample size was calculated based on a statistical formula by Cochran.[23] The sample size was based on a 0.3 effect size, $80 \%$ power, $95 \%$ confidence interval, a p-value of 0.05 , and $33 \% \mathrm{BA}$ site prevalence (2015) reported in the internal annual statistics. A systematic random sampling method was used, and the investigator selected 1 case out of $3\left(1^{\text {st }}, 4^{\text {th }}, 7^{\text {th }} \ldots\right)$ for the sample, while respecting the aforementioned inclusion and exclusion criteria.

\section{Measures}

The data collection tool included three sections. Section 1 was the demographic and obstetrical characteristics. Section 2 was the questionnaire that had been tested on the prevalence and risk factors of perinatal asphyxia at a hospital in Nigeria.[9] The tool was received on 3 September 2016 via email and adapted with permission to the Rwandan context and the conceptual framework of this study.

The following modifications were made to the tool. Two items were removed, the participant's name, and one item used for booking or non-booking status needed in Nigeria. Some items were added by the investigator, such as, the number of antenatal care visits during the mother's pregnancy, maternal age, aspect of amniotic fluid, and initial help provided to newborns after birth concerning neonatal resuscitation. Also, more possibilities on the delivery site were added, as Dr. Ilah's tool only focused on hospital and home.

The data collection instrument had a total of 25 items: 1 item to identify the participants with NICU index number; 1 to determine the age of NICU neonates on admission; 1 to determine the prevalence of $\mathrm{BA} ; 2$ to record the APGAR scores (at one and five minutes); 18 items to assess BA risk factors (4 to assess the fetal factors; 4 to assess maternal factors; 2 to assess placental factors; 5 to assess labor and birth factors and 3 items to assess postnatal factors) and three items to assess BA outcomes. 
Section 1: Demographic and obstetrical characteristics. Maternal factors (age, gravida, ANC contacts, comorbidities).Neonatal factors (gestational age, gender, birth-weight, singleton or multiple births).

Section 2: Labor, birth, post-natal factors. Time of rupture of membranes (ROM) to birth (normal is $<18$ hours, or prolonged $>18$ hours), and the condition of amniotic fluid (meconium stained, malodorous, or clear fluid). Labor: none, normal ( $<14$ hours), prolonged $(>15$ hours). Mode of birth (vaginal or cesarean).Apgar score ( $\leq 5$ or $\geq 6$ ). Cry at 5 minutes (yes or no). Resuscitation (routine care, ventilated, or cardiac massage).

Section 3: NICU measured outcome. Neonate discharged home, referred to tertiary hospital, or demise.

The tool was pretested on 34 cases admitted in NICU in 2017 for feasibility purposes. The tool indicated a Cronbach's Alpha of 0.81 .

\section{Data collection}

After the university and the district hospital granted permission, the investigator reviewed the births recorded in the 2016 Registration book located in the NICU. The registration book became the sample frame, and from there every third neonate was selected to be included in the sample. Some information was retrieved from the registration book, but most was retrieved from the neonatal files located in another part of the hospital.

\section{Data analysis}

Data were analyzed using SPSS software version 20. Descriptive statistics were used to interpret and summarize variables. Chi-square test, or Fisher's exact test (used for sample size $<5$ in a cell) were used to test associations between variables and BA. A p-value $<0.05$ was considered statistically significant. Variables with p-value 0.05 were analyzed with logistic regression to identify independent $\mathrm{BA}$ risk factors.

\section{Ethical considerations}

Ethical approval from the University of Rwanda, College of Medicine and Health Sciences Institutional Review Board (IRB), and the district hospital was obtained before data collection. The investigator ensured the integrity and quality of the research. As a retrospective study informed consent was unnecessary, but confidentiality and anonymity of the NICU registration book and neonatal file content were respected.

\section{Results}

The maternal age mean was 27.2 years, with a range from 15 to 43 years. The majority of mothers were primigravidae $(50.6 \%)$, had $\leq 3 \mathrm{ANC}$ contacts, with term gestation $(83.6 \%)$, and a vaginal birth $(66.5 \%)$. Most of the births were singletons $(97.6 \%)$ and born with normal birth weight beteen 2500 ang $3999 \mathrm{gr}$ (85.6\%).

\section{Maternal age}

Neonates born to mothers under 18 years of age were equally distributed in $\mathrm{BA}$ and non-BA groups (50\%) (table 1). Neonates born to mothers aged 35 years of age or older were a third $(33.3 \%)$ of BA cases, compared to younger aged mothers with two thirds $(66.7 \%)$ with BA.

\section{Gravida}

Of the 163 neonates born to mothers who were gravida one, nearly half $(45.4 \%)$ had BA. Of the 137 neonates born to mothers who were gravida $2-4$, a third $(33.6 \%)$ had BA, while mothers gravida 5 or more were less $(15.7 \%)$. The gravida of the mothers was statistically significant $\left(X^{2}, 10.442, p=0.005\right)$, though the multigravida population in this sample population was small.

\section{Antenatal care visits}

The proportion of ANC visits showed that nearly half $(45.5 \%)$ attended $\geq 4$, while over a third $(36.8 \%)$ attended 3 ANC visits.

\section{Co-morbidities}

The analysis of co-morbidity factors (such as malaria, HIV, hypertension, diabetes), during pregnancy and or labor, showed BA in over one third $(39.6 \%)$ of healthy mothers, and over one quarter $(28 \%)$ of those with comorbidities.

Maternal age, ANC contacts, and maternal comorbidities had no statistical significance but may have clinical significance $(\mathrm{p}>0.05)$.

Table 1. Demographic characteristics $(n=340)$

\begin{tabular}{|c|c|c|c|}
\hline & \multirow[b]{2}{*}{ Total } & \multicolumn{2}{|c|}{ Birth Asphyxia n (\%) } \\
\hline & & Yes & No \\
\hline \multicolumn{4}{|l|}{ Maternal age $^{\wedge}$} \\
\hline $15-17$ & $4(1.3)$ & $2(50.0)$ & $2(50.0)$ \\
\hline $18-34$ & $270(88.3)$ & $104(38.5)$ & $166(61.5)$ \\
\hline$\geq 35$ & $30(9.9)$ & $10(33.3)$ & $20(66.7)$ \\
\hline \multicolumn{4}{|l|}{ Gravida $^{\wedge}$} \\
\hline 1 & $163(50.6)$ & $74(45.4)$ & $89(54.6)$ \\
\hline $2-4$ & $137(42.6)$ & $46(33.6)$ & $91(66.4)$ \\
\hline$\geq 5$ & $22(6.8)$ & $3(13.6)$ & $19(86.4)$ \\
\hline \multicolumn{4}{|l|}{$\mathrm{ANC}_{\text {visits }}{ }^{\wedge}$} \\
\hline 3 & $114(78.0)$ & $42(36.8)$ & $72(63.2)$ \\
\hline$\geq 4$ & $33(22.0)$ & $15(45.5)$ & $18(54.5)$ \\
\hline \multicolumn{4}{|c|}{ Co-morbidities ${ }^{\wedge}$} \\
\hline Healthy mothers & $260(84)$ & $103(39.6)$ & $157(60.4)$ \\
\hline Mothers with & $50(1)(0)$ & $14(200)$ & \\
\hline \multicolumn{4}{|l|}{ Gestation } \\
\hline Near term & $43(12.6)$ & $10(23.3)$ & $33(76.7)$ \\
\hline Term & $284(83.6)$ & $116(40.8)$ & $168(59.2)$ \\
\hline Post-term & $13(3.8)$ & $9(69.2)$ & $4(30.8)$ \\
\hline \multicolumn{4}{|l|}{ Birth type } \\
\hline Vaginal & $226(66.5)$ & $107(47.3)$ & $119(52.7)$ \\
\hline Cesarean & $114(33.5)$ & $28(24.6)$ & $86(75.4)$ \\
\hline \multicolumn{4}{|c|}{ Neonatal gender } \\
\hline Female & $148(43.5)$ & $58(39.2)$ & $90(60.8)$ \\
\hline Male & $192(56.5)$ & $77(40.1)$ & $115(59.9)$ \\
\hline \multicolumn{4}{|l|}{ Birth weight } \\
\hline $1500-2499$ & $31(9.1)$ & $8(25.8)$ & $23(74.2)$ \\
\hline $2500-3999$ & $291(85.6)$ & $126(43.3)$ & $165(56.7)$ \\
\hline $4000-5450$ & $18(5.3)$ & $1(5.6)$ & $17(94.4)$ \\
\hline
\end{tabular}

^missing data: maternal age $\mathrm{n}=304$, gravida $\mathrm{n}=322$, ANC $\mathrm{n}=147$, co-morbidities $\mathrm{n}=310$.

Main co-morbidities included; HIV 24(7.7\%) and malaria $13\left(4.2^{\circ}\right.$ diabetes $3(1 \%)$, maternal fever $3(1.0 \%)$

There were 135 neonates out of 340 with $\mathrm{BA}$ in the NICU at the hospital in Kigali during 2016, giving a prevalence of $39.7 \%$ which is almost $40 \%$. (figure 1 ) 
Figure 1. Prevalence of BA in 2016

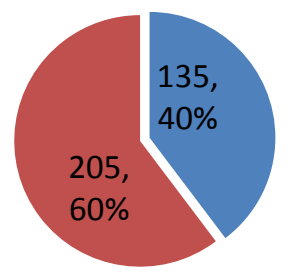

Birth Asphyxia Yes

Birth Asphyxia No

\section{Birth weight}

Of those infants that had BA, a quarter (25.8\%) weighed $\leq 2499$ grams, and nearly a half $(43.3 \%)$ were normal weight 2500-3999 grams (table 2). The normal birth weight category was statistically associated with BA ( $p=0.013)$. However, nearly all $(94.4 \%)$ 4000-gram neonates or more did not have BA.

\section{Gestation}

Of those infants that had BA, nearly a quarter $(23.3 \%)$ were near term, and more $(40.8 \%)$ were at term. Of the 13 post-term infants, over two thirds $(69.2 \%)$ had BA.

\section{Gravida}

Of the newborns with BA, nearly half (45.4\%) were born to primigravida mothers, a third $(33.6 \%)$ were gravida $2-4$, a smaller proportion (13.6\%) to gravida 5 or more. The parity of the mothers was statistically significant $(p=0.005)$. Maternal age, co-morbidities, ANC visits were not statistically significant for BA $(\mathrm{p}>0.05)$.

Table 2. Maternal Demographics and Obstetrical

\section{Characteristics $(n=340)$}

\begin{tabular}{|c|c|c|c|c|c|c|}
\hline \multirow[t]{2}{*}{ Variable } & \multicolumn{2}{|c|}{ Birth Asphyxia n (\%) } & \multirow{2}{*}{$\begin{array}{l}\mathrm{X}^{2} / \mathrm{F} \\
\text { Test }\end{array}$} & \multirow[t]{2}{*}{ OR } & \multirow[t]{2}{*}{$95 \% \mathrm{Cl}$} & \multirow[t]{2}{*}{$\mathrm{p}$} \\
\hline & Yes & No & & & & \\
\hline Birth weight & & & $14.00^{\mathrm{F}}$ & & & \\
\hline $1500-2499$ & $8(25.8)$ & $23(74.2)$ & & 5.91 & $0.67-51.86$ & 0.109 \\
\hline 2500-3999 & $126(43.3)$ & $165(56.7)$ & & 12.98 & $1.71-98.85$ & $0.013^{*}$ \\
\hline $4000-5450$ & $1(5.6)$ & $17(94.4)$ & & 1 & & \\
\hline Gestation & & & $9.75^{\mathrm{a}}$ & & & \\
\hline Near term & $10(23.3)$ & $33(76.7)$ & & 1 & & \\
\hline Term & $116(40.8)$ & $168(59.2)$ & & 2.28 & $1.08-4.81$ & $0.002 *$ \\
\hline Postterm & $9(69.2)$ & $4(30.8)$ & & 0.31 & $0.09-1.02$ & 0.054 \\
\hline Gravida $^{\wedge}$ & & & $10.44^{\mathrm{a}}$ & & & \\
\hline 1 & $74(45.4)$ & $89(54.6)$ & & 1 & & \\
\hline $2-4$ & $46(33.6)$ & $91(66.4)$ & & 3.20 & $0.90-11.38$ & 0.072 \\
\hline 5 & $3(13.6)$ & $19(86.4)$ & & 5.27 & $1.50-18.49$ & $0.010^{*}$ \\
\hline
\end{tabular}

$\mathrm{X}^{2 \& a}$ Chi-Square; F\&F Fisher exact test; OR Odds ratio; CI Confidence interval; ${ }^{*} \mathrm{p} \leq 0.05$.

${ }^{\wedge}$ Missing data: gravida $\mathrm{n}=322$.

\section{Amniotic fluid factors}

Of the neonates born to mothers with prolonged ROM (>18 hours), almost a quarter $(24.6 \%)$ had BA, whereas three quarters $(75.4 \%)$ did not have prolonged ROM (table 3). The duration of membrane rupture in BA was significant $(\mathrm{p}=0.049)$. Nearly a quarter $(20.8 \%)$ of mothers of neonates with BA had clear amniotic fluid, whereas nearly a half $(48.3 \%)$ had meconium stained or malodorous fluid. The association of amniotic fluid status and BA was significant $(\mathrm{p}=0.000)$.

\section{Labor and birth}

Over a third $(37.2 \%)$ of the BA neonates were born from labor of normal duration ( $<18$ hours), whereas nearly a quarter $(20 \%)$ had prolonged labor ( $>18$ hours). The duration of labor in BA was significant $(p=0.041)$. Nearly half $(47.3 \%)$ of the BA neonates were born vaginally, whereas a quarter $(24.6 \%)$ had a cesarean birth. The mode of delivery in BA was significant $(\mathrm{p}=0.000)$.

\section{Postnatal factors}

The majority of $\mathrm{BA}$ was diagnosed in neonates with an Apgar score $\leq 5$ at 5 minutes (92.4\%), and did not cry in the first 5 minutes after birth $(79.4 \%)$. Of the BA neonates, nearly a quarter $(21.8 \%)$ received only the first steps of newborn resuscitation (dry stimulation suctioning), over two thirds (68\%) were ventilated, and over three quarters $(78.9 \%)$ were resuscitated with cardiac massage. All three factors were statistically significant for BA: 5-minute Apgar score $(\mathrm{p}=0.000)$, crying $(\mathrm{p}=0.000)$, and resuscitation $(\mathrm{p}=0.000)$.

\section{Signs of BA}

The main signs of BA during the first 24 hours after birth included seizures, hypotonic status/limp/floppy, and hyperactivity. Of the neonates that had seizures; three quarters $(76.3 \%)$ had BA, though nearly a quarter $(23.7 \%)$ did not have BA. Of the neonates with hypotonic status; two thirds $(66.7 \%)$ had BA, a limited proportion $(10 \%)$ did not have BA, and the rest were unknown $(23.3 \%)$. Of the neonates with hyperactivity, nearly half $(45.5 \%)$ had $\mathrm{BA}$ and over half $(54.5 \%)$ did not have BA. 
Table 3. Labor and Birth Factors Associated with BA

\begin{tabular}{|c|c|c|c|c|c|c|}
\hline \multirow[t]{2}{*}{ Variable } & \multicolumn{2}{|c|}{ Birth Asphyxia n (\%) } & \multirow{2}{*}{$\begin{array}{l}\mathrm{X}^{2} / \mathrm{F} \\
\text { Test }\end{array}$} & \multirow[t]{2}{*}{ OR } & \multirow[t]{2}{*}{$95 \% \mathrm{CI}$} & \multirow[t]{2}{*}{$\mathrm{p}$} \\
\hline & Yes & No & & & & \\
\hline$\overline{\text { ROM}^{\wedge}}$ & & & $3.88^{\mathrm{a}}$ & & & \\
\hline Normal duration & $72(38.1)$ & $117(61.9)$ & & 1.885 & $1.00-3.56$ & $0.049 *$ \\
\hline Prolonged & $16(24.6)$ & $49(75.4)$ & & 1 & & \\
\hline Amniotic fluid ${ }^{\wedge}$ & & & $16.11^{\mathrm{a}}$ & & & \\
\hline Stained/malodorous & $71(48.3)$ & $76(51.7)$ & & 3.562 & $1.88-6.75$ & $0.000 *$ \\
\hline Clear & $16(20.8)$ & $61(79.2)$ & & 1 & & \\
\hline Labor duration^^${ }^{\wedge}$ & & & $6.38^{\mathrm{F}}$ & & & \\
\hline No labor & $2(11.1)$ & $16(88.9)$ & & 1 & & \\
\hline Normal (<18h) & $70(37.2)$ & $118(62.8)$ & & 4.746 & $1.06-2.26$ & $0.042 *$ \\
\hline Prolonged (>18h) & $3(20.0)$ & $12(80.0)$ & & 2 & $0.29-1.91$ & 0.484 \\
\hline Mode of birth & & & $16.43^{\mathrm{a}}$ & & & \\
\hline Vaginal & $107(47.3)$ & $119(52.7)$ & & 2.762 & $1.68-4.55$ & $0.000^{*}$ \\
\hline Cesarean & $28(24.6)$ & $86(75.4)$ & & 1 & & \\
\hline APGAR (5 mins) & & & $13.22^{\mathrm{a}}$ & & & \\
\hline 5 & $73(92.4)$ & $0(0.0)$ & & 5.186 & $2.00-13.42$ & $0.001 *$ \\
\hline 6 & $61(70.1)$ & $26(29.9)$ & & 1 & & \\
\hline $\operatorname{Cry}(5 \text { mins })^{\wedge}$ & & & $93.03^{\mathrm{a}}$ & & & \\
\hline None & $50(79.4)$ & $13(20.6)$ & & 38.811 & $16.27-92.60$ & $0.000 *$ \\
\hline Cried & $11(9.0)$ & $111(91.0)$ & & 1 & & \\
\hline Resuscitation $^{\wedge}$ & & & $66.03^{a}$ & & & \\
\hline Routine care & $36(21.8)$ & $129(78.2)$ & & 1 & & \\
\hline Ventilated & $68(68.0)$ & $32(32.0)$ & & 2.030 & $1.49-2.68$ & $0.003^{*}$ \\
\hline Cardiac massage & $15(78.9)$ & $4(21.1)$ & & 2.598 & $1.60-4.29$ & $0.003^{*}$ \\
\hline
\end{tabular}

$\mathrm{X}^{2 \& a}$ Chi-Square; F*F Fisher exact test; OR Odds ratio; CI Confidence interval; ${ }^{*} \mathrm{p} \leq 0.05$; mins $=$ minutes; $\mathrm{h}=$ hour.

${ }^{\wedge}$ Missing data: $\operatorname{ROM} n=254$, amniotic fluid $n=224$, labor duration $n=221$, cry at 5 mins $n=185$, resuscitation $n=284$.

\section{Outcomes of BA neonates}

Neonates were hospitalized in the NICU between $0-38$ days, and the birth outcomes are presented in table 4. The mean hospital stay was 6.2 days (SD:0.93), 315 neonates $(92.6 \%)$ received an authorized discharge, and $2(0.6 \%)$ were referred at tertiary level care. Twenty three $(6.7 \%)$ neonates died, and of those $10(43.5 \%)$ in the first 24 hours after birth, and 13(56.5\%) after 24 hours.

Table 4. Outcomes of BA $(n=340)$
There was an equal female to male ratio of 1:1 in the sample, though BA male neonates were more likely to die $(57.1 \%)$ than female. In the NICU population, all deaths occurred in the first 21 days of life, with a neonatal mortality rate of $23 / 340$ (6.8\%); BA specific mortality was $87 \%(20 / 23)$, with a case fatality rate of $14.8 \%$ (20/135). The death in BA cases occurred within the first 24 hours in 9 cases $(45 \%)$ and 11 cases (55\%) between 1-21 days.

\begin{tabular}{lllll}
\hline Birth Asphyxia & $\begin{array}{l}\text { Discharged } \\
\mathbf{n}(\mathbf{\%})\end{array}$ & $\begin{array}{l}\text { Referred } \\
\mathbf{n}(\mathbf{\%})\end{array}$ & $\begin{array}{l}\text { Demise (<24h) } \\
\mathbf{n}(\mathbf{\%})\end{array}$ & $\begin{array}{l}\text { Demise (>24h*) } \\
\mathbf{n}(\mathbf{\%})\end{array}$ \\
\hline Yes & $113(35.9)$ & $2(100)$ & $9(90)$ & $11(84.6)$ \\
No & $202(64.1)$ & $0(0.0)$ & $1(10)$ & $2(15.4)$ \\
Total & $315(100)$ & $2(100)$ & $10(100)$ & $13(100)$ \\
\hline
\end{tabular}

*Demise occurred between 1 and 21 days. h=hours

\section{Discussion}

The purpose of the study was to assess the prevalence and identify possible risk factors of BA in a NICU in the capital city of Rwanda. BA was diagnosed in 135 out of 340 babies admitted to the NICU study site in 2016, giving a prevalence of $39.7 \%$, which was higher than the internal records for 2015 (33\%). This rate was notably higher than the $30.1 \%$ reported in Nigeria, [9] and 29.3\% reported in Tanzania.[24] In Ethiopia, the prevalence rate was $3.1 \%$, but only three quarters $(81.5 \%)$ of the records had complete documentation, so the final rate 
was reduced to $2.5 \%$.[25] In contrast, another study in Pakistan had a higher prevalence (51.25\%).[4]

Risk factors associated with BA included mainly healthy term neonates from mothers who attended the recommended four ANC visits, had a normal length of labor and normal duration of ROM, a normal spontaneous vaginal delivery, and normal birth weight (2500-3999 g). Such findings are considered normal factors, but in this study were associated with BA. These findings highlight the need for vigilant monitoring of every labor and birth, by a truly skilled birth attendant, as BA is not limited to previously known risk factors and can occur at any time, especially during the intrapartum and immediate postnatal period. Two other studies address normal term neonates and BA prevalence. $[9,26$,

Though poor ANC attendance has been associated with poor pregnancy outcomes, $[27,28]$ in this study population, women who had $<4$ ANC visits had a lower $\mathrm{BA}$ rate $(36.8 \%)$ than those attending 4 ANC visits who had a higher $\mathrm{BA}$ rate $(45.5 \%)$. Therefore, the higher number of ANC visits offered no apparent protective effect. This factor may speak more to the quality of these four visits and not quantity; indeed, four ANC visits does not provide enough opportunities to identify specific risk factors, nor provide adequate treatment. In fact, since the time of this study the WHO has increased the frequency of ANC from four to at least eight "contacts," emphasizing the need for quality contacts verses visits, and implying a hands-on approach, such as measuring blood pressure and fundal height.[29]

Quality ANC should never be minimized as an essential means of assuring a healthy mother and neonate. Perhaps the women who attended more frequently had more health concerns or issues. In Kenya and Uganda, and about 30 other countries worldwide, pregnant women keep their own ANC record in a "Mother \& Child Health Booklet,'[30] which provides vital health education for the mother/family and an accurate record of the quantity and quality of ANC. The booklet contains essential evidenced-based health information across the continuum of maternal and neonatal care, with a provision for recording all maternal interventions during the perinatal period, and recommended interventions and care from birth to five years of age for the child.

While prolonged ROM was not a statistically significant factor, neonates born in meconium-stained fluid were four times more likely to have $\mathrm{BA}$, compared to those born with clear amniotic fluid (OR 3.562, CI 1.881-6.745, $\mathrm{p}=0.000$ ). Passing meconium into the amniotic fluid has long been considered an indicator of fetal distress and should alert the birth attendant for the possible need for neonatal assistance during the transition to extrauterine life. Meconium-stained amniotic fluid was a noted associated factor for BA in several other studies. $[19,26,31,32]$ However, skilled birth attendants need to know that routine suctioning of neonates is no longer recommended as evidence-based practice as it may increase the risk of $\mathrm{BA}$ and should be avoided. $[5,13]$

Mothers' gravida was statistically significant $\left(X^{2}, 10.442\right.$, $\mathrm{p}=0.005)$; mothers with $\geq 5$ births had an increased risk of BA. However, the number of women with high gravida in this population was small $(6 \%)$, so findings should be viewed cautiously. Though another study with a larger sample $(43 \%)$ did find that higher parity ( $\geq 4)$ was a risk factor for BA.[34] Mode of birth was a risk factor for BA. Two-thirds of our population was born via vaginal birth $(66.5 \%)$, and nearly half $(47.3 \%)$ had BA. One-third was born via cesarean $(33.5 \%)$, and a quarter $(24.6 \%)$ had BA. Similarly, a study in Pakistan found that $44 \%$ of vaginal births, $32 \%$ of cesarean births, and $23 \%$ of instrument-assisted delivery had neonates with BA.[34] Postnatal associated factors showed that most of the $\mathrm{BA}$ neonates were of normal weight and male, but there was no statistical significance for gender compared to other studies. $[9,35]$

Three other factors were statistically significant for BA; 5-minute Apgar score, crying, and resuscitation $(\mathrm{p}=0.000 ; \mathrm{p}=0.000 ; \mathrm{p}=0.000$, respectively) need consideration. The perinatal medical community has known for years that the accuracy of Apgar scores was often questionable because of discrepancies between delivery room staff in the way they performed the test and documented the results. This situation is no different today, as in many resource-limited counties, APGAR scoring can be inconsistent, inaccurate and not recorded systematically.[36] When interpreting these findings, we must ask if some neonates did not receive the appropriate level of neonatal resuscitation that they needed due to inaccurate Apgar scoring.

Compared to neonates who received routine care and/or the first step of resuscitation (dry stimulation suctioning), BA was observed more often in neonates who received advanced steps of neonatal resuscitation. $\mathrm{BA}$ was two times more likely in neonates who were ventilated, (OR 2.030, CI 1.49-2.68, $\mathrm{p}=0.003$ ); and two and half times more likely in neonates who received cardiac massage (OR 2.598, CI 1.60-4.29, $\mathrm{P}=0.003$ ). Indeed, these findings point to the need for accurate APGAR scoring. Since the Apgar score is subjective with a high level of inter-observer variability; $[36,37]$ an observational study on the timing and accuracy of each of the five Apgar elements in Rwanda is recommended.

These findings also clearly demonstrate the need for ongoing training and recertification in Helping Babies Breathe $(\mathrm{HBB})$, and programs such as Essential Care 
for Every Baby (ECEB), and Essential Care for Small Babies (ECSB). HBB has been shown to decrease neonatal mortality significantly. The WHO estimates that $5-10 \%$ of newborns will require some assistance to initiate respirations, including 3-6\% who require ventilation by a bag and mask.[13] Rwandan perinatal healthcare providers would benefit from annual refresher courses needed to increase their knowledge retention and prevent loss of skills learned in the initial training courses.[38] Currently the 50,000 Happy Birthdays programme is underway to train, equip and empower Rwandan midwives and nurses in HMS and HBB.

The HBB programs were developed to reduce neonatal mortality in resource-limited environments. Although the percentage of neonates that require advanced resuscitation techniques such as chest compressions and cardiac medications is small, given the severe consequences of $\mathrm{BA}$ there is a time and place when advanced training in a neonatal resuscitation program (NRP) should be instituted to prevent and/or minimize the effects of BA. The study site is a busy urban district maternity hospital with a large NICU. While HBB has been taught to most, if not all of their perinatal staff, there are few if any who are skilled and certified in NRP. The concern is that infants who require such efforts to survive would likely need ongoing support. However, such care could and should exist at the district hospital level.

As with any program whether basic $\mathrm{HBB}$ or NRP the need to have the necessary equipment and supplies at all times cannot be overstated. There needs to be clean clothes for drying and stimulating the newborn, a clock for APGAR scoring, and a functional neonatal bag and mask. For NRP, there needs to be adrenaline and epinephrine and oxygen, as well as syringes and needles, a laryngoscope, and appropriate size endotracheal tubes. The NRP guidelines for medication administration should be posted in each delivery room. Ideally, there should be at least two certified staff per full-scope resuscitation.

Concerning outcomes, BA cases presented with three main signs; seizures; limp/ floppy/ hypotonic status, and hyperactivity. These are key findings as seizures are reported to be more common in HIE grade II (moderate stage). In the first 24 hours, seizures were reported in $76.3 \%$ of $\mathrm{BA}$ neonates. A limp/floppy/ hypotonic status was present in $66.7 \%$ of $\mathrm{BA}$ neonates, while hyperactivity was present in $45.5 \%$. According to Sarnat and Sarnat HIE staging; Hyperactivity is the most dominant clinical sign in HIE grade I, whereas a limp/ floppy/ hypotonic status is more associated with a severe form of HIE (grade II or III).[39] Furthermore, seizures can be a predictor of permanent neurological damage.
BA neonates experienced prolonged NICU stays compared to non-BA babies. The mean number of days in the NICU was 7.6 (SD: 6.1) for BA babies while it was 5.3 (SD: 4.16) in babies without BA. The Sarnat staging system was not used in this study, but prolonged hospital stays, ranging from weeks to months, are known to be associated with Sarnat stages II and III.[39]

Three reasons were noted for the neonate leaving the NICU; authorized discharge, referral to a higher clinical setting, and death. In the NICU population, all deaths occurred in the first 21 days of life with a neonatal mortality rate of $23 / 340$ (6.8\%); BA specific mortality was $87 \%(20 / 23)$ with a case fatality rate of $14.8 \%$ (20/135). Comparing those findings to the $\mathrm{MOH}$ reports about causes of neonatal deaths, BA remains the leading cause of neonatal death. These findings are two times higher than noted in the Rwandan Annual Health Statistics Booklet (2014),[17] which means the $\mathrm{BA}$ rate has increased.

A recent study in Rwanda eluded that the proportion of preventable deaths was high among stillbirths and early neonatal deaths, suggesting much could be done to lower BA rates.[15] The survivors of BA experienced mild to severe neurological damage. The quality of life of these BA neonates discharged from the NICU is beyond the scope of this study.

\section{Limitations}

This retrospective study concerned only cases admitted to one NICU in Kigali in 2016 and therefore cannot be generalized to other areas. The BA prevalence may not represent an accurate statistic of BA, as some cases might have died and may not have been admitted to the NICU. The detailed management of BA and the longterm complications, family experience, quality of life of BA survivors is beyond the scope of this study.

The investigator had many challenges with incomplete, missing and inaccurate documentation in the NICU registers and neonatal files. Since it was a retrospective study, the amount of missing data was unavoidable, though the variables affected were identified in tables. The poor record keeping was problematic, e.g., the APGAR scoring was inconsistent, inaccurate and not recorded systematically in some cases. Another issue was trying to retrieve information on placental factors, as it was part of the conceptual framework, but not necessarily available in the file. Instead, we were able to focus on amniotic fluid factors, e.g., length of rupture of membranes and meconium stained fluid. Furthermore, the investigator found that the diagnosis of $\mathrm{BA}$ at the study site has many different names in French and English languages.

\section{Future research}

Studies are needed, both qualitative and quantitative to 
find out what happens to these children, and their families, as they negotiate the care of raising a special needs child.

\section{Conclusion}

The study findings show that BA was diagnosed in 135 out of 340 neonates admitted to the NICU, indicating a prevalence rate of $39.7 \%$ among neonates admitted to a specific NICU during 2016. This finding reveals a 6.7\% increase of $\mathrm{BA}$ prevalence than recorded the previous year at the same hospital. Several modifiable and nonmodifiable risk factors were identified. BA outcomes were found to be high mortality, early seizures, and prolonged hospital stay. Trained healthcare professionals could do more to lower the BA rate in Rwanda.

\section{Acknowledgments}

Thank you to Dr. Pamela Meharry for support with this publication through the writing retreats and manuscript development, and Dr. Sue Prullage for reviewing this manuscript.

This article is published open access under the Creative Commons Attribution-NonCommercial NoDerivatives (CC BY-NC-ND 4.0). People can copy and redistribute the article only for noncommercial purposes and as long as they give appropriate credit to the authors. They cannot distribute any modified material obtained by remixing, transforming or building upon this article. See https:// creativecommons.org/licenses/by-nc-nd/4.0/

\section{References}

1. UNICEF. UNICEF 2018 Neonatal mortality [Internet]. 2018. Available from: https://data. unicef.org/topic/child-survival/neonatalmortality/

2. WHO. 2018 Neonatal mortality data [Internet]. 2018. Available from: https://www.who.int/ gho/child_health/mortality/neonatal_text/en/

3. UNICEF. Every newborn 1 [Internet]. 2014. Available from: http://apps.who.int/iris/bitstream/ handle/10665/127938/9789241507448_eng. pdf?sequence $=1.2014$.

4. Aslam HM uhamma., Saleem S, Afzal R, Iqbal U, Saleem SM uhamma., Shaikh MW aqa. A, et al. "Risk factors of birth asphyxia." Ital J Pediatr. 2014;40:94.

5. Healthy Newborn Network. Helping Babies Breathe [Internet]. 2018. Available from: https://www.healthynewbornnetwork.org/ partner/helping-babies-breathe/

6. Rhoda NR, Chb MB, Neonatology C, Velaphi S, Chb MB, Gebhardt GS, et al. Reducing neonatal deaths in South Africa : Progress and challenges. 2018;3(March):9-16.
7. Muhammed G, Kibria A, Burrowes V, Choudhury A, Sharmeen A, Ghosh S, et al. Determinants of early neonatal mortality in Afghanistan: an analysis of the Demographic and Health Survey 2015. 2018;1-12.

8. Sloan NL, Storey A, Fasawe O, Yakubu J, McCrystal K, Wiwa O, et al. Advancing Survival in Nigeria: A Pre-post Evaluation of an Integrated Maternal and Neonatal Health Program. Matern Child Health J [Internet]. 2018;22(7):986-97. Available from: http:// dx.doi.org/10.1007/s10995-018-2476-3

9. Ilah BG, Aminu MS, Musa A, Adelakun MB. Prevalence and Risk Factors for Perinatal Asphyxia as Seen at a Specialist Hospital in Gusau, Nigeria. 2015;2(2):1-6.

10. Tabassum F, Rizvi A, Ariff S, Soofi S, Bhutta ZA. Risk Factors Associated with Birth Asphyxia in Rural District Matiari, Pakistan : A Case-Control Study. 2014;(December):1430-41.

11. Mohamed MM. Mechanisms of Birth Asphyxia and a Novel Resuscitation Strategy. Biological and Environmental Sciences. 2014.

12. Masemo A, Maimuna A, Khatib M, Marie G, Dnp W, Rende EK, et al. Helping Babies Breathe implementation in Zanzibar, Tanzania. 2017;(April):1-7.

13. American Academy of Pediatrics. Helping Babies Breathe. Provider guide $2^{\text {nd }}$ edition 2016.

14. Kak L. Helping Babies Breathe. Lessons Learned Guiding the way forward. 2017; Available from: https://surviveandthrive.org/ about/Documents/Survive Thrive 5 year report FINAL.pdf

15. Musafili A, Persson L-åke, Baribwira C, Påfs J, Mulindwa PA, Essén B. Case review of perinatal deaths at hospitals in Kigali, Rwanda : perinatal audit with application of a three-delays analysis. 2017;1-13.

16. Ministry Of Health. Rwanda Annual Health Statistics Booklet Table of contents [Internet]. 2012. Available from: http://www.moh.gov. rw/fileadmin/templates/HMIS_Docs/MOH_ Booklet_2012.pdf

17. Ministry Of Health. Rwanda Annual Health Statistics Booklet 2014. Kigali-Rwanda;

18. Walana W, Ks AE, Naafu B, Aruk E, E VK, Kampo S, et al. Clinics in Mother and Child Health Pattern, Causes and Treatment Outcomes of Neonatal Admission in the Tamale Teaching Hospital. 2016;13(4). 
19. Demisse AG, Alemu F, Gizaw MA, Tigabu Z. Patterns of admission and factors associated with neonatal mortality among neonates admitted to the neonatal intensive care unit of University of Gondar Hospital, Northwest Ethiopia. Pediatric health, medicine, and therapeutics. 2017;8:57.

20. Hedstrom A, Ryman T, Otai C, Nyonyintono J, Mcadams RM, Lester D. Demographics, clinical characteristics and neonatal outcomes in a rural Ugandan NICU. 2015;1-9.

21. Mduma E, Svensen E, Perlman J. Birth Asphyxia: A Major Cause of Early Neonatal Mortality in a Tanzanian Rural Hospital. 2012;

22. Magadi M, DiamondI. Conceptual framework Pathways Of The Determinants Of Unfavourable Birth Outcomes In Kenya. J Biomed Sci. 2004;36(2):153-76.

23. Israel GD. Determining Sample Size 1. 1992;(November):1-5.

24. UNICEF. Tanzania. Maternal and Neonatal Health Disparities. 2015; Available from: https://data.unicef.org/wp-content/uploads/ country_profiles/United Republic of Tanzania/ country profile_TZA.pdf

25. Ibrahim NA, Muhye A, Abdulie S. Prevalence of Birth Asphyxia and Associated Factors among Neonates Delivered in Dilchora Referral Hospital, in Dire Dawa, Eastern Ethiopia Clinics in Mother and Child Health. 2017;14(4). Available from: https://pdfs.semanticscholar. org/f204/4daffa47457ebff52a6e3928871c 1b865764.pdf

26. Deviibhai K Vadher Surendranagr DrMehul D Sindha DrKetanGadhavi. A study of etiology, onset and clinical manifestations of neonatal seizures. Pediatrics. 2017;(4):276-80.

27. Jongh TE De, Urganci IG, Allen E, Zhu NJ. Integration of antenatal care services with health programmes in low - and middle - income countries : a systematic review. 2016;6(1).

28. Adanikin AI, Awoleke JO. Clinical suspicion, management and outcome of intrapartum fetal distress in a public hospital with limited advanced fetal surveillance. 2017;7058.

29. WHO Recommendations on Antenatal Care for a Positive Pregnancy Experience: Summary Highlights and Key Messages from the World Health Organization' s 2016 Global Recommendations for Routine Antenatal Care.
2018;10(January):1-10.

30. WHO. 29 WHO recommendation on antenatal care contact schedules [Internet]. 2018. Available from: https://extranet.who.int/rhl/ topics/improving-health-system-performance/ who-recommendation-antenatal-care-contactschedules

31. Mudany MA, Sirengo M, Rutherford GW, Mwangi M, Nganga LW, Gichangi A. Enhancing Maternal and Child Health using a Combined Mother \& Child Health Booklet in Kenya. 2015;(September):442-7.

32. Tasew H, Zemicheal M, Teklay G, Mariye T, Ayele E. Risk factors of birth asphyxia among newborns in public hospitals of Central. BMC Res Notes [Internet]. 2018;1-7. Available from: https://doi.org/10.1186/s13104-018-3611-3

33. Prakash R. Clinical Profile and Neurobehaviour at Discharge of Term Neonates with Perinatal Asphyxia - A Prospective Observational Study. 2016;3(10):3073-6.

34. Kiyani AN, Khushdil A, Ehsan A. Perinatal Factors Leading to Birth Asphyxia among Term Newborns in a Tertiary Care Hospital. 2014;24(5):637-42.

35. Nair CC, Madhu GN, Srinivasa S, Mn M. Clinical profile and outcome of perinatal asphyxia in a tertiary care center. 2015;19:9-12.

36. Bharti B, Bharti S. A review of the Apgar score indicated that contextualization was required within the contemporary perinatal and neonatal care framework in different settings. 2005;58:121-9.

37. Ahearne CE, Boylan GB, Murray DM, Ahearne CE, Boylan GB, Murray DM, et al. update. 2016;5(1):67-75.

38. Essén B, Persson L-åke, Musafili A, Ess B. Evaluating Helping Babies Breathe: training for healthcare workers at hospitals in Rwanda Evaluating Helping Babies Breathe: training for healthcare workers at hospitals in Rwanda. 2012; (November).

39. Ministry of Health. The Republic of Rwanda. National Neonatal Care Protocol. 2014;(September). 